\title{
High-field noise in metallic diffusive conductors
}

\author{
Frederick Green $\dagger$ and Mukunda P Dasł \\ $\dagger$ GaAs IC Prototyping Facility, CSIRO Telecommunications and Industrial Physics, PO Box 76, Epping NSW 1710, Australia \\ $\ddagger$ Department of Theoretical Physics, Research School of Physical Sciences and Engineering, The Australian National \\ University, Canberra ACT 0200, Australia
}

\begin{abstract}
We analyze high-field current fluctuations in degenerate conductors by mapping the electronic Fermi-liquid correlations at equilibrium to their semi-classical non-equilibrium form. Our resulting Boltzmann description is applicable to diffusive mesoscopic wires. We derive a non-equilibrium connection between thermal fluctuations of the current and resistive dissipation. In the weak-field limit this is the canonical fluctuation-dissipation theorem. Away from equilibrium, the connection enables explicit calculation of the excess "hot-electron" contribution to the thermal spectrum. We show that excess thermal noise is strongly inhibited by Pauli exclusion. This behaviour is generic to the semi-classical metallic regime.
\end{abstract}

73.50. $\mathrm{Td}, 72.10 . \mathrm{Bg}, 73.50 . \mathrm{Fq}$

\section{INTRODUCTION}

In this paper we address a technologically important open problem: non-equilibrium noise in strongly driven degenerate conductors. Nano-fabrication has made possible a variety of refined measurements of transport and noise, for many different structures at sub-micron dimensions [1] 8]. Alongside the experiments there has been much theoretical activity [9].

A thriving topic is the behaviour of current fluctuations in diffusive wires. Typically, this concerns structures shorter than the bulk inelastic mean free path but still much longer than that for elastic scattering. They are in an operating region where randomness of the carrier motion prevails. This is our regime of interest.

Two theories have come to the fore as methods of choice for describing mesoscopic transport. These are inherently weak-field models, predicated upon exclusively linear forms of transport analysis. One technique (Landauer-Büttiker) is based on coherent quantum transmission [9, 17]. This has been adapted to fluctuations and noise by Khlus [10], Lesovik [11], Beenakker and Büttiker [12,13], Martin and Landauer [14], and many others [9, 17]. Another approach (Boltzmann-Langevin) uses stochastic transport equations [9], reduced to a diffusive model [18]. The same phenomenology has since been applied to fluctuations and noise by Nagaev 15 and de Jong and Beenakker [9, 16].

Although these mesoscopic-noise methodologies are markedly distinct, both agree on their paradigm. They view a mesoscopic wire as a random assembly of individual elastic scatterers, in a bath of free carriers whose propagation, impeded by the scatterers, must be regarded as strictly diffusive and must be calculated as such [18]. For an exhaustive survey of diffusive noise theories we cite the recent review of Blanter and Büttiker [17] as well as the earlier one of de Jong and Beenakker [9].

The leading diffusive methods for noise also share a number of difficulties. Not least among these is the issue of conformity with the fluctuation structure of charged Fermi liquids 19]. This and other basic problems are analyzed in detail in references 20] and [21].

Clean, truly first-principles solutions certainly exist for non-equilibrium noise. Monte Carlo simulations are very well established, as witness those of the Lecce group [22], even if high-field Monte Carlo is still rare for metals [23, 24]. In the non-degenerate case an analytical, self-contained and computable theory of noise has been formulated by Korman and Mayergoyz [25]. Their approach is strictly kinetic and free of superfluous phenomenological props. In philosophy it is akin to Green-function models for fluctuations, such as Stanton and Wilkins' 26,27.

In developing a microscopically consistent account of noise, there are cogent reasons to stay within the traditional kinetic canons rather than embrace novel diffusive doctrines. The chief reason, perhaps, is sheer technological need; device designers can scarcely afford to be hobbled by phenomenologies whose congenital linearity denies any access to the vital high-field region. The small scale of modern device structures means that they are routinely driven into non-linear response [28].

To illustrate this point we estimate the range of validity for linear diffusion. In the weak-field limit the Einstein relation [18,20], or drift-diffusion equivalence, underpins diffusive transport. Roughly speaking, drift-diffusion equivalence breaks down (and linear diffusive transport with it) when the energy gained in drift mediated by inelastic scattering exceeds the energy scale for diffusion mediated by elastic processes. In a short metallic wire this means that $(e V / L) \min \left\{L, L_{\mathrm{in}}\right\} \gtrsim \hbar v_{\mathrm{F}} / L_{\mathrm{el}}$, where $V$ is the driving voltage, $L$ is the sample length and $L_{\mathrm{in}}$ is the (bulk) 
inelastic mean free path at the Fermi surface. Similarly $L_{\mathrm{el}}$ is the elastic mean free path $\left(v_{\mathrm{F}}\right.$ is the Fermi velocity). Note that for $L_{\mathrm{in}}>L$ the effective inelastic path becomes the sample length, since dissipation in the bounding leads is dominant.

For a typical mesoscopic silver wire of electron density $6 \times 10^{22} \mathrm{~cm}^{-3}$, the low-temperature transport parameters are [1] $L_{\mathrm{in}}=1 \mathrm{~cm} \gg L=30 \mu \mathrm{m}$ and $L_{\mathrm{el}}=50 \mathrm{~nm}$. The threshold voltage is then $V \gtrsim 40 \mathrm{mV}$ for the breakdown of the diffusive regime. In a two-dimensional mesoscopic channel at density $2 \times 10^{11} \mathrm{~cm}^{-2}$ the threshold is appreciably lower, with [1] $L=17 \mu \mathrm{m}, L_{\mathrm{in}}=6 \mu \mathrm{m}$ and $L_{\mathrm{el}}=1.4 \mu \mathrm{m}$ giving $V \gtrsim 0.25 \mathrm{mV}$. This shows how readily mesoscopic devices, particularly low-dimensional ones, can enter the non-linear regime beyond diffusive theory.

The present is the first of three studies covering the essential formalism for non-equilibrium noise, the action of Coulomb correlations in non-uniform systems [29] and finally the kinetic description of shot noise [30]. Throughout, we follow one overarching principle. It is that a consistent model of non-equilibrium fluctuations will conform to basic properties of the electron gas in a natural way, if and only if such a model is grounded explicitly in the theory of the electronic Fermi liquid.

To require that a kinetic description of fluctuations and noise respect fundamental conservation laws in equilibrium, is to place a unifying constraint on its low- and high-field forms together. Our aim is to catalogue all the physical consequences of this assertion. A viable kinetic model will necessarily recover the fluctuation-dissipation relation [31], but it must also contain the equally fundamental conserving sum rules 32].

Much of the authoritative literature on diffusive noise theory, if not all of it, prefers to make a virtue of its heavy dependence on drift-diffusion equivalence and the fluctuation-dissipation theorem, while remaining oblivious to every other basic sum-rule requirement. Such an understanding is too scanty. It is the full physics of Fermi liquids which governs their fluctuations, a fact which cannot simply be ignored.

An established tool for scattering-dominated noise in degenerate conductors is the semi-classical Green-function approach [26,27,33 35]. We take it beyond its well-understood role in time-dependent response, by proving that the dynamical Green function also governs the structure of the adiabatic (steady-state) fluctuations. In turn, these determine the mean initial strength of the time-dependent fluctuations.

The non-equilibrium adiabatic fluctuations are exact closed functionals of their equilibrium form. This offers the key to practical and flexible calculations over a wide range of useful non-perturbative collision models. (Here the detailed classical studies of Stanton and Wilkins [26,27] show the way.) Such problems are entirely out of range for the Boltzmann-Langevin models, of wide currency but quite narrow practicality for conductors in strong driving fields [26], with strong internal interactions [36.

In section 2 we present a wholly conventional Boltzmann description of carrier fluctuations in non-uniform metallic systems, down to the same mesoscopic range accessible to alternative (diffusive) models [9, 17. We demonstrate the quantitative connection between fluctuations and power dissipation well out of equilibrium. In the weak-field limit, this connection is the canonical fluctuation-dissipation theorem (FDT). At high fields, it describes the hot-electron contribution to current noise. This lets us calculate, in section 3, the excess thermal spectrum, which is strongly suppressed in a degenerate system. In section 4 we sum up, and preview the two forthcoming works.

\section{THEORY}

The theoretical discussion is in six parts. We begin by formulating the transport problem as a direct mapping of the electron Fermi liquid to its non-equilibrium steady state. Next we describe the steady-state fluctuations, after which we discuss time dependence, then the dynamic fluctuations and their formal connection with the steady state. This produces a self-contained expression for the current-current fluctuation, which determines thermal noise. Last, we analyze the connection between fluctuations and dissipation in the non-equilibrium region.

\section{A. Transport Model}

The semi-classical Boltzmann transport equation for the electron distribution function $f_{\alpha}(t) \equiv f_{s}(\mathbf{r}, \mathbf{k}, t)$ is

$$
\left[\frac{\partial}{\partial t}+\mathbf{v}_{\mathbf{k} s} \cdot \frac{\partial}{\partial \mathbf{r}}-\frac{e \mathbf{E}(\mathbf{r}, t)}{\hbar} \cdot \frac{\partial}{\partial \mathbf{k}}\right] f_{\alpha}(t)=-\sum_{\alpha^{\prime}}\left[W_{\alpha^{\prime} \alpha}\left(1-f_{\alpha^{\prime}}\right) f_{\alpha}-W_{\alpha \alpha^{\prime}}\left(1-f_{\alpha}\right) f_{\alpha^{\prime}}\right] .
$$

Label $\alpha=\{\mathbf{k}, s, \mathbf{r}\}$ denotes a point in single-particle phase space, while sub-label $s$ indexes both the discrete sub-bands (or valleys) of a multi-level system and the spin state. The system is acted upon by the total field $\mathbf{E}(\mathbf{r}, t)$. We study single-particle scattering, with a rate $W_{\alpha \alpha^{\prime}} \equiv \delta\left(\mathbf{r}-\mathbf{r}^{\prime}\right) W_{s s^{\prime}}\left(\mathbf{k}, \mathbf{k}^{\prime} ; \mathbf{r}\right)$ that is local in real space, independent of the 
driving field, and that satisfies detailed balance: $W_{\alpha^{\prime} \alpha}\left(1-f_{\alpha^{\prime}}^{\mathrm{eq}}\right) f_{\alpha}^{\mathrm{eq}}=W_{\alpha \alpha^{\prime}}\left(1-f_{\alpha}^{\mathrm{eq}}\right) f_{\alpha^{\prime}}^{\mathrm{eq}}$ where $f_{\alpha}^{\mathrm{eq}}$ is the equilibrium distribution. In a system with $\nu$ dimensions, we make the following correspondence for the identity operator:

$$
\delta_{\alpha \alpha^{\prime}} \equiv \delta_{s s^{\prime}}\left\{\frac{\delta_{\mathbf{r r}^{\prime}}}{\Omega(\mathbf{r})}\right\}\left\{\Omega(\mathbf{r}) \delta_{\mathbf{k k}^{\prime}}\right\} \longleftrightarrow \delta_{s s^{\prime}} \delta\left(\mathbf{r}-\mathbf{r}^{\prime}\right)(2 \pi)^{\nu} \delta\left(\mathbf{k}-\mathbf{k}^{\prime}\right)
$$

The volume $\Omega(\mathbf{r})$ of a local cell in real space becomes the measure for spatial integration, while its inverse defines the scaling in wave-vector space for the local bands $\{\mathbf{k}, s\}$.

The first step is to construct the steady-state solution $f_{\alpha} \equiv f_{\alpha}(t \rightarrow \infty)$ explicitly from $f^{\text {eq }}$, which satisfies the equilibrium form of equation (11):

$$
\left[\mathbf{v}_{\mathbf{k} s} \cdot \frac{\partial}{\partial \mathbf{r}}-\frac{e \mathbf{E}_{0}(\mathbf{r})}{\hbar} \cdot \frac{\partial}{\partial \mathbf{k}}\right] f_{\alpha}^{\mathrm{eq}}=0=-\sum_{\alpha^{\prime}}\left[W_{\alpha^{\prime} \alpha}\left(1-f_{\alpha^{\prime}}^{\mathrm{eq}}\right) f_{\alpha}^{\mathrm{eq}}-W_{\alpha \alpha^{\prime}}\left(1-f_{\alpha}^{\mathrm{eq}}\right) f_{\alpha^{\prime}}^{\mathrm{eq}}\right] .
$$

The internal field $\mathbf{E}_{0}(\mathbf{r})$ is defined in the absence of a driving field. The quantities $f^{\text {eq }}$ and $\mathbf{E}_{0}$ are linked self-consistently by the usual constitutive relations, the first being the Poisson equation

$$
\frac{\partial}{\partial \mathbf{r}} \cdot \epsilon \mathbf{E}_{0}=-4 \pi e\left(\left\langle f^{\mathrm{eq}}(\mathbf{r})\right\rangle-n^{+}(\mathbf{r})\right)
$$

in terms of the dielectric constant $\epsilon(\mathbf{r})$, the electron density $\left\langle f^{\mathrm{eq}}(\mathbf{r})\right\rangle \equiv \Omega(\mathbf{r})^{-1} \sum_{\mathbf{k}, s} f_{\alpha}^{\text {eq }}$, and the positive background density $n^{+}(\mathbf{r})$, which is taken to be independent of the driving field [37. Normalization to the total particle number is $\sum_{\mathbf{r}} \Omega(\mathbf{r})\left\langle f^{\text {eq }}(\mathbf{r})\right\rangle=N$. The second relation is the form of the equilibrium function itself,

$$
f_{\alpha}^{\mathrm{eq}}=\left[1+\exp \left(\frac{\varepsilon_{\alpha}-\phi_{\alpha}}{k_{B} T}\right)\right]^{-1}
$$

at temperature $T$. The conduction-band energy $\varepsilon_{\alpha}=\varepsilon_{s}(\mathbf{k} ; \mathbf{r})$ may have structural parameters that depend on position implicitly. The locally defined Fermi level $\phi_{\alpha}=\mu-V_{0}(\mathbf{r})$ is the difference of the global chemical potential $\mu$ and the electrostatic potential $V_{0}(\mathbf{r})$, whose gradient is $e \mathbf{E}_{0}(\mathbf{r})$.

Define the difference function $g_{\alpha}=f_{\alpha}-f_{\alpha}^{\text {eq. }}$. From each side of equation (11) in the steady state, subtract its equilibrium counterpart [38]. We obtain

$$
\begin{aligned}
\mathbf{v}_{\mathbf{k} s} \cdot \frac{\partial g_{\alpha}}{\partial \mathbf{r}}-\frac{e \mathbf{E}(\mathbf{r})}{\hbar} \cdot \frac{\partial g_{\alpha}}{\partial \mathbf{k}}= & \frac{e\left(\mathbf{E}-\mathbf{E}_{0}\right)}{\hbar} \cdot \frac{\partial f_{\alpha}^{\mathrm{eq}}}{\partial \mathbf{k}}-\sum_{\alpha^{\prime}}\left(W_{\alpha^{\prime} \alpha} g_{\alpha}-W_{\alpha \alpha^{\prime}} g_{\alpha^{\prime}}\right) \\
& +\sum_{\alpha^{\prime}}\left(W_{\alpha^{\prime} \alpha}-W_{\alpha \alpha^{\prime}}\right)\left(f_{\alpha^{\prime}}^{\mathrm{eq}} g_{\alpha}+g_{\alpha^{\prime}} f_{\alpha}^{\mathrm{eq}}+g_{\alpha} g_{\alpha^{\prime}}\right)
\end{aligned}
$$

The solutions to equations (2) and (4) are determined by the asymptotic conditions in the source and drain reservoirs, be it at equilibrium or with an external electromotive force. The active region includes the carriers within source and drain terminals out to several screening lengths. This means that local fields are negligible at the interfaces with the reservoirs; in practice, one shorts out the fields so that $\mathbf{E}(\mathbf{r})=\mathbf{E}_{0}(\mathbf{r})=\mathbf{0}$ beyond these boundaries. Then Gauss's theorem implies that the system remains globally neutral:

$$
\sum_{\mathbf{r}} \Omega(\mathbf{r})\langle g(\mathbf{r})\rangle \equiv \sum_{\alpha} g_{\alpha}=0 .
$$

Recast equation (4) as an integro-differential equation. The inhomogeneous term on its right-hand side generates the explicit dependence on the equilibrium state of the system:

$$
\sum_{\alpha^{\prime}} B\left[W^{A} f\right]_{\alpha \alpha^{\prime}} g_{\alpha^{\prime}}=\frac{e \widetilde{\mathbf{E}}(\mathbf{r})}{\hbar} \cdot \frac{\partial f_{\alpha}^{\mathrm{eq}}}{\partial \mathbf{k}}+\sum_{\alpha^{\prime}} W_{\alpha \alpha^{\prime}}^{A} g_{\alpha^{\prime}} g_{\alpha} .
$$

The net non-equilibrium field $\mathbf{E}-\mathbf{E}_{0}$ is represented here as $\widetilde{\mathbf{E}} \equiv \mathbf{E}_{\text {ext }}+\mathbf{E}_{\text {ind }}$, consisting of $\mathbf{E}_{\text {ext }}(\mathbf{r})$, the externally applied field [39], plus the local induced response $\mathbf{E}_{\mathrm{ind}}(\mathbf{r})$. The linearized Boltzmann operator $B\left[W^{A} f\right]$ is

$$
B\left[W^{A} f\right]_{\alpha \alpha^{\prime}}=\delta_{\alpha \alpha^{\prime}}\left[\mathbf{v}_{\mathbf{k}^{\prime} s^{\prime}} \cdot \frac{\partial}{\partial \mathbf{r}^{\prime}}-\frac{e \mathbf{E}\left(\mathbf{r}^{\prime}\right)}{\hbar} \cdot \frac{\partial}{\partial \mathbf{k}^{\prime}}+\sum_{\beta}\left(W_{\beta \alpha^{\prime}}-W_{\beta \alpha^{\prime}}^{A} f_{\beta}\right)\right]-W_{\alpha \alpha^{\prime}}+W_{\alpha \alpha^{\prime}}^{A} f_{\alpha},
$$


with $W_{\alpha \alpha^{\prime}}^{A}=W_{\alpha \alpha^{\prime}}-W_{\alpha^{\prime} \alpha}$. Note that $W^{A}=0$ if the scattering is elastic or if a linear approximation (such as Drude) replaces the explicit Boltzmann collision term.

If it is to represent the physical solution, $g$ must vanish with $\widetilde{\mathbf{E}}$ in the equilibrium limit. This is guaranteed by the Poisson equation for the induced field,

$$
\frac{\partial}{\partial \mathbf{r}} \cdot \epsilon \mathbf{E}_{\text {ind }}=-4 \pi e\left(\langle f(\mathbf{r})\rangle-\left\langle f^{\mathrm{eq}}(\mathbf{r})\right\rangle\right)=-4 \pi e\langle g(\mathbf{r})\rangle .
$$

\section{B. Steady-State Response}

To calculate the adiabatic response of the system about its steady non-equilibrium operating point, we introduce the propagator 40 ]

$$
G_{\alpha \alpha^{\prime}} \stackrel{\text { def }}{=} \frac{\delta g_{\alpha}}{\delta f_{\alpha^{\prime}}^{\text {eq }}}
$$

with a global constraint following directly from equation (5):

$$
\sum_{\alpha} G_{\alpha \alpha^{\prime}}=0 \text { for all } \alpha^{\prime}
$$

The equation for $G$ is derived by taking variations on both sides of equation (雨):

$$
\sum_{\beta} B\left[W^{A} f\right]_{\alpha \beta} G_{\beta \alpha^{\prime}}=\delta_{\alpha \alpha^{\prime}}\left[\frac{e \widetilde{\mathbf{E}}\left(\mathbf{r}^{\prime}\right)}{\hbar} \cdot \frac{\partial}{\partial \mathbf{k}^{\prime}}+\sum_{\beta} W_{\beta \alpha^{\prime}}^{A} g_{\beta}\right]-W_{\alpha \alpha^{\prime}}^{A} g_{\alpha} .
$$

The variation is restricted by excluding the reaction of the local fields $\mathbf{E}_{0}(\mathbf{r})$ and $\mathbf{E}(\mathbf{r})$. This means that $G$ is a response function free of Coulomb screening. Here we treat the electrons as an effectively neutral Fermi liquid. In our second paper we will describe the complete fluctuation structure, with Coulomb effects [29].

All of the steady-state fluctuation properties induced by the thermal background will be specified in terms of $G$ and the equilibrium two-body fluctuation. This consists of the "proper" electron-hole pair correlation in its static long-wavelength limit (up to a normalization factor). In the free-electron approximation [32, that correlation is

$$
\lim _{q \ll k_{\mathrm{F}}}\left[\lim _{\omega \rightarrow 0}\left(\frac{f_{s}^{\mathrm{eq}}(\mathbf{r}, \mathbf{k}-\mathbf{q} / 2)-f_{s}^{\mathrm{eq}}(\mathbf{r}, \mathbf{k}+\mathbf{q} / 2)}{\hbar \omega-\varepsilon_{s}(\mathbf{k}+\mathbf{q} / 2 ; \mathbf{r})+\varepsilon_{s}(\mathbf{k}-\mathbf{q} / 2 ; \mathbf{r})}\right)\right]=-\frac{\delta f_{\alpha}^{\mathrm{eq}}}{\delta \varepsilon_{\alpha}}=\frac{\delta f_{\alpha}^{\mathrm{eq}}}{\delta \phi_{\alpha}}
$$

where the net momentum transfer becomes negligible relative to the Fermi wave number $k_{\mathrm{F}}$. The formal statisticalmechanical definition of the (mean square) occupation-number fluctuation $\Delta f^{\text {eq }}$ takes it as the variation of the occupancy $f^{\text {eq }}$ of equation (3b) with respect to the electro-chemical potential and normalized to the thermal energy, keeping $T$ and the local volume $\Omega(\mathbf{r})$ fixed. That is,

$$
\Delta f_{\alpha}^{\mathrm{eq}} \equiv k_{\mathrm{B}} T \frac{\delta f_{\alpha}^{\mathrm{eq}}}{\delta \phi_{\alpha}}
$$

It is easy to derive the free-electron form of the equilibrium fluctuation:

$$
\Delta f_{\alpha}^{\mathrm{eq}}=f_{\alpha}^{\mathrm{eq}}\left(1-f_{\alpha}^{\mathrm{eq}}\right) .
$$

When there are strong exchange-correlation interactions the two-body correlation, equation (11a), is renormalized by a coefficient that depends on the Landau quasi-particle parameters. This coefficient carries through in $\Delta f^{\mathrm{eq}}$. In the present model we neglect exchange-correlation effects. This is a valid approximation in dense degenerate systems [32].

Before discussing the non-equilibrium fluctuations we comment on the crucial contrast between the quantumFermi-liquid origin of equation (11) and the widespread Boltzmann-Langevin approach, which is essentially classical. Equation (11a) describes an elementary and kinematically coupled electron-hole excitation [a fact that is particularly obvious in the particle-hole structure of the energy denominator, $\left.\hbar \omega-\varepsilon_{s}(\mathbf{k}+\mathbf{q} / 2 ; \mathbf{r})+\varepsilon_{s}(\mathbf{k}-\mathbf{q} / 2 ; \mathbf{r})\right]$. Its form is determined by the same quantum dynamical equation whose semi-classical limit is the Boltzmann equation itself [41]. 
The physical character of a polarized excitation demands its representation as a self-contained entity. In thermodynamic terms, stochasticity attaches to the spontaneous generation of electron-hole pairs in the system and not to their electron and hole constituents independently (as if pairwise charge balance were of no real consequence). By its nature, a pair fluctuation cannot be decomposed ad hoc into two stochastically unlinked single-particle factors. The Boltzmann-Langevin approach, on the other hand, is tantamount to such a notional decomposition [31. Neither natural nor necessary for the right description of the elementary fluctuations in a charged Fermi liquid, it is eminently dispensable.

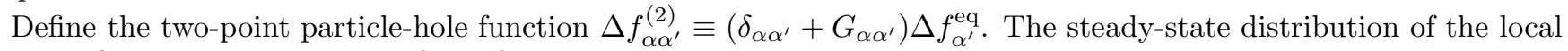
number fluctuation is the sum of all of the two-body terms:

$$
\Delta f_{\alpha}=\sum_{\alpha^{\prime}} \Delta f_{\alpha \alpha^{\prime}}^{(2)}=\Delta f_{\alpha}^{\mathrm{eq}}+\sum_{\alpha^{\prime}} G_{\alpha \alpha^{\prime}} \Delta f_{\alpha^{\prime}}^{\mathrm{eq}} \text { for all } \alpha .
$$

Once the explicit solution for $G$ is obtained, the behaviour of $\Delta f$ is known completely. This non-equilibrium fluctuation satisfies the linearized steady-state Boltzmann equation:

$$
\sum_{\beta} B\left[W^{A} f\right]_{\alpha \beta} \Delta f_{\beta}=0
$$

We see that the solution to this equation is manifestly a linear functional of its equilibrium counterpart. Jointly, equations (12) and (13) mean that any scaling behaviour exhibited by the fluctuations at equilibrium must also be exhibited by the actual fluctuations for the non-equilibrium problem. That is the direct result of local equilibrium in the asymptotic leads, and of overall neutrality in the system made up of conductor plus leads.

The proportionality of all thermally induced noise to ambient temperature $T$ is inevitable in the degenerate limit. This has implications for understanding shot noise in metallic conductors 20,21,30. Shot noise never scales with $T$. We comment further on the scaling issue in the later sections.

One may compare the analysis in terms of $G$ and $\Delta f^{(2)}$ with the equal-time correlator introduced by Gantsevich et al. 34]. The equal-time correlator is itself a hierarchical functional of other correlators (such as the current fluctuations), whose solutions are unknown a priori and which must be closed by force, either by truncation or by an ad hoc heuristic device such as Boltzmann-Langevin. This makes for a less-than-tractable computational scheme, at least beyond a narrow repertoire of special limits (linear response; classical particles; weak non-uniformity). In particular, a calculable strategy for degenerate non-equilibrium fluctuations, based on the equal-time correlator, has yet to be demonstrated.

By contrast, we show below that $G$ is explicitly determined by the dynamical Green function for the linearized Boltzmann equation [see equation (30)]. As pointed out by Stanton [27], the Boltzmann-Green functions are much more straighforward to compute for a wide range of collision models. This ease of calculation extends to $G$ and hence to $\Delta f^{(2)}$, which provides the initial conditions for a naturally closed dynamical solution.

Global charge neutrality requires that the total fluctuation strength over the sample, $\Delta N=\sum_{\mathbf{r}} \Omega(\mathbf{r})\langle\Delta f(\mathbf{r})\rangle$, be conserved. This constrains not only the steady-state but also the time-dependent fluctuations.

\section{Time Dependence}

Calculation of the dynamic response requires the time-dependent Green function [33]

$$
R_{\alpha \alpha^{\prime}}\left(t-t^{\prime}\right) \stackrel{\text { def }}{=} \theta\left(t-t^{\prime}\right) \frac{\delta f_{\alpha}(t)}{\delta f_{\alpha^{\prime}}\left(t^{\prime}\right)},
$$

with initial value $R_{\alpha \alpha^{\prime}}(0)=\delta_{\alpha \alpha^{\prime}}$. As with $G$, the variation is restricted. The linearized Boltzmann equation satisfied by $R\left(t-t^{\prime}\right)$ is derived from equation (11) and takes the form

$$
\sum_{\beta}\left\{\delta_{\alpha \beta} \frac{\partial}{\partial t}+B\left[W^{A} f\right]_{\alpha \beta}\right\} R_{\beta \alpha^{\prime}}\left(t-t^{\prime}\right)=\delta\left(t-t^{\prime}\right) \delta_{\alpha \alpha^{\prime}}
$$

Summation over $\alpha$ on both sides of this equation gives zero contribution from $\sum_{\alpha} \sum_{\beta} B\left[W^{A} f\right]_{\alpha \beta} R_{\beta \alpha^{\prime}}\left(t-t^{\prime}\right)$. Subsequent integration over $t$ leads to conservation of normalization [33]:

$$
\sum_{\alpha} R_{\alpha \alpha^{\prime}}\left(t-t^{\prime}\right)=\theta\left(t-t^{\prime}\right)
$$


The time-dependent propagator is a two-point correlation. It tracks the history of a fluctuation of unit strength, created in state $\alpha^{\prime}$ at time $t^{\prime}$. The fluctuation strength in state $\alpha$, at the later time $t$, is $R_{\alpha \alpha^{\prime}}\left(t-t^{\prime}\right)$. In the long-time limit equation (15) goes to the steady-state equation (13) independently of $\alpha^{\prime}$, so that $R_{\alpha \alpha^{\prime}}(t \rightarrow \infty) \propto \Delta f_{\alpha}$. Together with equation (16) this gives [33]

$$
R_{\alpha \alpha^{\prime}}(t \rightarrow \infty)=\frac{\Delta f_{\alpha}}{\Delta N}
$$

All of the time-dependent fluctuation properties induced by the thermal background are specified in terms of $R$ and the steady-state non-equilibrium fluctuation $\Delta f$. From the dynamical particle-hole propagator [34], that is $\Delta f_{\alpha \alpha^{\prime}}^{(2)}(t) \equiv R_{\alpha \alpha^{\prime}}(t) \Delta f_{\alpha^{\prime}}$, one constructs the lowest-order moment

$$
\Delta f_{\alpha}(t)=\sum_{\alpha^{\prime}} \Delta f_{\alpha \alpha^{\prime}}^{(2)}(t)
$$

in analogy with equation (12). Equation (15), with its adjoint [33], implies that $\Delta f_{\alpha}(t)=\Delta f_{\alpha}$ for $t>0$. Thus the intrinsic time dependence of $\Delta f^{(2)}(t)$ is not revealed through this quantity [12]. Equation (16) ensures constancy of the total fluctuation strength: $\sum_{\mathbf{r}} \Omega(\mathbf{r})\langle\Delta f(\mathbf{r}, t)\rangle=\Delta N$ for $t>0$.

\section{Dynamic Correlations}

We move to the frequency domain. An important outcome is the quantitative link between fluctuations and resistive power dissipation in the non-equilibrium regime. This requires expressing both the difference function $g$ and the adiabatic propagator $G$ directly in terms of the dynamical Green function. The Fourier transform $\mathrm{R}(\omega)=\int d t e^{i \omega t} R(t)$ of the retarded time-dependent Green function satisfies

$$
\sum_{\beta}\left\{B\left[W^{A} f\right]_{\alpha \beta}-i \omega \delta_{\alpha \beta}\right\} \mathrm{R}_{\beta \alpha^{\prime}}(\omega)=\delta_{\alpha \alpha^{\prime}}
$$

showing that $R(\omega)$ is the resolvent for the linearized Boltzmann operator of equation (7). From equation (16), the global condition on the resolvent is

$$
\sum_{\alpha} \mathrm{R}_{\alpha \alpha^{\prime}}(\omega)=-\frac{1}{i(\omega+i \eta)} \quad\left(\eta \rightarrow 0^{+}\right) .
$$

At face value this does not match the corresponding criterion for $G$, equation (9). To solve equation (10) for the steady-state propagator explicitly in terms of the dynamic one, we follow Kogan and Shul'man [33] in introducing the intrinsically correlated part of $\mathrm{R}(\omega)$. This is

$$
\mathrm{C}_{\alpha \alpha^{\prime}}(\omega)=\mathrm{R}_{\alpha \alpha^{\prime}}(\omega)+\frac{1}{i(\omega+i \eta)} \frac{\Delta f_{\alpha}}{\Delta N} .
$$

Once the long-time adiabatic term is removed, $\mathrm{C}(\omega)$ conveys the purely transient response of the system. It satisfies a pair of identities [33]. First, the Fourier transform of the relation $\Delta f(t)=\theta(t) \Delta f$ translates to

$$
\sum_{\alpha^{\prime}} \mathrm{C}_{\alpha \alpha^{\prime}}(\omega) \Delta f_{\alpha^{\prime}}=0 \text { for all } \alpha,
$$

while equation (20) leads to

$$
\sum_{\alpha} \mathrm{C}_{\alpha \alpha^{\prime}}(\omega)=0 \text { for all } \alpha^{\prime} .
$$

The latter parallels the constraint on $G$. Like $\mathrm{R}(\omega)$, the correlated propagator is analytic in the upper half-plane $\operatorname{Im}\{\omega\}>0$, and satisfies the Kramers-Krönig dispersion relations. Unlike $\mathrm{R}(\omega)$, however, $\mathrm{C}(\omega)$ is regular for $\omega \rightarrow 0$.

We now obtain $g$ and $G$ in terms of the correlated dynamical response. Consider the equation

$$
\sum_{\alpha^{\prime}}\left\{B\left[W^{A} f\right]_{\alpha \alpha^{\prime}}-i \omega \delta_{\alpha \alpha^{\prime}}\right\} g_{\alpha^{\prime}}(\omega)=\frac{e \widetilde{\mathbf{E}}(\mathbf{r})}{\hbar} \cdot \frac{\partial f_{\alpha}^{\mathrm{eq}}}{\partial \mathbf{k}}+\sum_{\alpha^{\prime}} g_{\alpha} W_{\alpha \alpha^{\prime}}^{A} g_{\alpha^{\prime}}
$$


inversion with the resolvent yields

$$
\mathrm{g}_{\alpha}(\omega)=\sum_{\alpha^{\prime}} \mathrm{C}_{\alpha \alpha^{\prime}}(\omega) \frac{e \widetilde{\mathbf{E}}\left(\mathbf{r}^{\prime}\right)}{\hbar} \cdot \frac{\partial f_{\alpha^{\prime}}^{\mathrm{eq}}}{\partial \mathbf{k}^{\prime}}+\sum_{\alpha^{\prime} \beta} \mathrm{C}_{\alpha \alpha^{\prime}}(\omega) g_{\alpha^{\prime}} W_{\alpha^{\prime} \beta}^{A} g_{\beta}
$$

The dominant low-frequency component of $\mathrm{R}(\omega)$ does not contribute to the right-hand side of this equation. In the first term it results in a decoupling of the summation over $\alpha^{\prime}$, yielding zero because $\partial f_{\alpha^{\prime}}^{\text {eq }} / \partial \mathbf{k}^{\prime}$ is odd in $\mathbf{k}^{\prime}$; in the second term, decoupling means that the double summation over $\alpha^{\prime}$ and $\beta$ vanishes by antisymmetry. In the static limit equation (24) becomes the inhomogeneous equation (6); moreover equation (23) means that $\mathrm{g}(\omega=0)$ satisfies equation (5), the sum rule for $g$. Therefore $g=\mathrm{g}(0)$, or

$$
g_{\alpha}=\sum_{\alpha^{\prime}} \mathrm{C}_{\alpha \alpha^{\prime}}(0) \frac{e \widetilde{\mathbf{E}}\left(\mathbf{r}^{\prime}\right)}{\hbar} \cdot \frac{\partial f_{\alpha^{\prime}}^{\mathrm{eq}}}{\partial \mathbf{k}^{\prime}}+\sum_{\alpha^{\prime} \beta} \mathrm{C}_{\alpha \alpha^{\prime}}(0) g_{\alpha^{\prime}} W_{\alpha^{\prime} \beta}^{A} g_{\beta} .
$$

This identity is central to the fluctuation-dissipation theorem.

In models with symmetric scattering, $W^{A}$ is zero and the adiabatic Green function assumes a simple form on varying both sides of equation (26):

$$
G_{\alpha \alpha^{\prime}}=\mathrm{C}_{\alpha \alpha^{\prime}}(0) \frac{e \widetilde{\mathbf{E}}\left(\mathbf{r}^{\prime}\right)}{\hbar} \cdot \frac{\partial}{\partial \mathbf{k}^{\prime}}
$$

More generally, an analysis similar to that for $\mathrm{g}(\omega)$ can be used directly for the adiabatic propagator. Introduce the operator $\mathrm{G}(\omega)$, defined to satisfy the dynamic extension of equation (10),

$$
\sum_{\beta}\left\{B\left[W^{A} f\right]_{\alpha \beta}-i \omega \delta_{\alpha \beta}\right\} \mathrm{G}_{\beta \alpha^{\prime}}(\omega)=\delta_{\alpha \alpha^{\prime}}\left[\frac{e \widetilde{\mathbf{E}}\left(\mathbf{r}^{\prime}\right)}{\hbar} \cdot \frac{\partial}{\partial \mathbf{k}^{\prime}}+\sum_{\beta} W_{\beta \alpha^{\prime}}^{A} g_{\beta}\right]-W_{\alpha \alpha^{\prime}}^{A} g_{\alpha} .
$$

This has the solution

$$
\mathrm{G}_{\alpha \alpha^{\prime}}(\omega)=\mathrm{C}_{\alpha \alpha^{\prime}}(\omega) \frac{e \widetilde{\mathbf{E}}\left(\mathbf{r}^{\prime}\right)}{\hbar} \cdot \frac{\partial}{\partial \mathbf{k}^{\prime}}-\sum_{\beta}\left(\mathrm{C}_{\alpha \alpha^{\prime}}(\omega)-\mathrm{C}_{\alpha \beta}(\omega)\right) W_{\alpha^{\prime} \beta}^{A} g_{\beta}
$$

In the first term on the right-hand side, the low-frequency component of $\mathrm{R}(\omega)$ makes no contribution after decoupling because the physical distributions $F_{\alpha}$ on which $\mathrm{G}(\omega)$ operates vanish sufficiently fast that $\sum_{\mathbf{k}} \partial F_{\alpha} / \partial \mathbf{k}=\mathbf{0}$. In the second right-hand term the uncorrelated parts of $\mathrm{R}_{\alpha \alpha^{\prime}}(\omega)$ and $\mathrm{R}_{\alpha \beta}(\omega)$ cancel directly. We conclude as before that

$$
G_{\alpha \alpha^{\prime}}=\mathrm{C}_{\alpha \alpha^{\prime}}(0) \frac{e \widetilde{\mathbf{E}}\left(\mathbf{r}^{\prime}\right)}{\hbar} \cdot \frac{\partial}{\partial \mathbf{k}^{\prime}}-\sum_{\beta}\left(\mathrm{C}_{\alpha \alpha^{\prime}}(0)-\mathrm{C}_{\alpha \beta}(0)\right) W_{\alpha^{\prime} \beta}^{A} g_{\beta}
$$

This is a crucial result. It shows (i) that the adiabatic structure of the steady state, through $G$, is of one piece with the correlated dynamic response (the result of causality and global charge neutrality), and (ii) that the non-equilibrium correlation structure evolves expressly out of the equilibrium state, through the specific functional form of $G \Delta f^{\mathrm{eq}}$.

We have proved that this non-perturbative kinetic description of fluctuations is self-contained, given its conventional set of assumptions and boundary conditions. The kinetic formalism has inherent predictive power. Hence, extraneous phenomenologies are not needed to make it viable. This is in sharp distinction to the diffusive Boltzmann-Langevin viewpoint 31.

\section{E. Spectral Density}

The vehicle for the physics of current noise is the velocity auto-correlation. It is a two-point distribution in real space, built on the correlated part of the two-particle fluctuation $\Delta \mathrm{f}_{\alpha \alpha^{\prime}}^{(2)}(\omega)=\mathrm{R}_{\alpha \alpha^{\prime}}(\omega) \Delta f_{\alpha^{\prime}}$. Following Gantsevich, Gurevich, and Katilius [34] it is a double sum over the kinematic states:

$$
\left\langle\left\langle\mathbf{v} \mathbf{v}^{\prime} \Delta \mathrm{f}^{(2)}\left(\mathbf{r}, \mathbf{r}^{\prime} ; \omega\right)\right\rangle\right\rangle_{\mathrm{c}}^{\prime} \stackrel{\text { def }}{=} \frac{1}{\Omega(\mathbf{r})} \sum_{\mathbf{k}, s} \frac{1}{\Omega\left(\mathbf{r}^{\prime}\right)} \sum_{\mathbf{k}^{\prime}, s^{\prime}} \mathbf{v}_{\mathbf{k} s} \operatorname{Re}\left\{\mathrm{C}_{\alpha \alpha^{\prime}}(\omega)\right\} \mathbf{v}_{\mathbf{k}^{\prime} s^{\prime}} \Delta f_{\alpha^{\prime}}
$$


Its physical meaning is the following. At any time, the system in steady state has a fluctuation background that is fed by spontaneous energy exchanges with the (equilibrium) thermal bath. The average strength of the fluctuations is fixed by the distribution $\Delta f$. The elementary modes making up this background are long-wavelength electron-hole excitations; these are given by $\Delta \mathrm{f}^{(2)}(\omega)$. The pair excitations are not themselves dynamically stable. Their transient evolution is determined by the propagator $\mathrm{C}(\omega)$ acting upon the ensemble-averaged background source, $\Delta f$. Finally, the velocity-velocity correlation for the pair process is obtained by attaching velocity operators at the start and end of the electron-hole excitations, and summing over states [32].

This approach to auto-correlations makes straightforward, and completely standard, use of the Boltzmann [34] and Fermi-liquid [32] theories. Our particular contribution is to have given an explicit recipe for computing the steadystate form of $\Delta f$ semi-classically, by analyzing the underlying adiabatic propagator $G$. Practical calculations should thereby become easier for degenerate systems at high driving fields.

The one-point object derived from equation (31),

$$
S_{f}(\mathbf{r}, \omega)=e^{2} \sum_{\mathbf{r}^{\prime}} \Omega\left(\mathbf{r}^{\prime}\right)\left\langle\left\langle(\widetilde{\mathbf{E}}(\mathbf{r}) \cdot \mathbf{v})\left(\widetilde{\mathbf{E}}\left(\mathbf{r}^{\prime}\right) \cdot \mathbf{v}^{\prime}\right) \Delta \mathbf{f}^{(2)}\left(\mathbf{r}, \mathbf{r}^{\prime} ; \omega\right)\right\rangle_{\mathrm{c}}^{\prime},\right.
$$

measures the local effect of fluctuations that are spread throughout the system. Formally it is the auto-correlation function of the power transferred from field to carriers, an inherently volume-distributed property that is represented here in terms of a locally defined spectral density. $S_{f}$ is closely related to the thermally induced current noise, integrated over the entire structure. In the weak-field limit it satisfies the FDT.

The two-point velocity correlator

$$
\left\langle\left\langle\mathbf{v} \mathbf{v}^{\prime} \Delta \mathrm{f}^{(2)}\left(\mathbf{r}, \mathbf{r}^{\prime} ; \omega\right)\right\rangle_{\mathrm{c}}^{\prime} / \Delta N\right.
$$

which is the response to a unit change of total particle number (and which does not scale with $T$ ), should provide the direct basis for shot-noise calculations across distances $\left|\mathbf{r}-\mathbf{r}^{\prime}\right|$ comparable to the mean free path. It is natural to ask how shot noise fits into the framework of equation (32). Within semi-classical kinetics, the short answer is that shot noise cannot be encompassed by the generic spectrum for thermal noise. For, as we have rigorously shown, all thermal fluctuations are required to scale with $T$ in the strongly degenerate (metallic) regime. Shot noise, on the other hand, has no such scaling. Therefore, whatever the kinetic description of shot noise may be, it is impossible for it to exhibit the smooth physical "cross-over" into thermal noise that is the primary feature of every diffusive model [31].

Our approach to the kinetics of mesoscopic shot noise is explored in reference [30]. In reference [20] we propose a quite specific experimental test of our theory. The new predictions made there are in stark contradistinction to the diffusive ones.

\section{F. Fluctuation and Dissipation}

The fluctuation-dissipation relation near equilibrium ties the spectral density of the thermal current fluctuations to the dissipative effects of the steady current in the system. However, dissipation by itself does not exhaust the physics of this sum rule. There are non-linear terms, negligible in linear response, that dominate the high-field behaviour of the noise [26,35]. In view of this, it is imperative to reveal the precise nature and action of these terms. We do so.

The resolvent property of $\mathrm{R}(\omega)$ provides a formal link between the steady-state (one-body) solution $g$ and the dynamical (two-body) fluctuation $\Delta \mathrm{f}^{(2)}$ at the semi-classical level. Taken to its equilibrium limit this becomes the familiar theorem. The connection is made in two steps. Consider the kinematic identity

$$
\frac{\partial f_{\alpha}^{\mathrm{eq}}}{\partial \mathbf{k}}=-\frac{\hbar}{k_{\mathrm{B}} T} \mathbf{v}_{\mathbf{k} s} \Delta f_{\alpha}^{\mathrm{eq}}
$$

and apply it to the leading term on the right-hand side of equation (26). The result is

$$
g_{\alpha}=-\frac{e}{k_{\mathrm{B}} T} \sum_{\alpha^{\prime}} \mathrm{C}_{\alpha \alpha^{\prime}}(0)(\widetilde{\mathbf{E}} \cdot \mathbf{v})_{\alpha^{\prime}} \Delta f_{\alpha^{\prime}}^{\mathrm{eq}}+h_{\alpha},
$$

in which $h_{\alpha}=\sum_{\alpha^{\prime} \beta} \mathrm{C}_{\alpha \alpha^{\prime}}(0) g_{\alpha^{\prime}} W_{\alpha^{\prime} \beta}^{A} g_{\beta}$. Evaluation of the current density according to $\mathbf{J}(\mathbf{r})=-e\langle\mathbf{v} g\rangle$, means that the power density $P(\mathbf{r})=\widetilde{\mathbf{E}}(\mathbf{r}) \cdot \mathbf{J}(\mathbf{r})$ for Joule heating can be written as

$$
P(\mathbf{r})=\frac{e^{2}}{k_{\mathrm{B}} T} \frac{1}{\Omega(\mathbf{r})} \sum_{\mathbf{k}, s} \sum_{\alpha^{\prime}}(\widetilde{\mathbf{E}} \cdot \mathbf{v})_{\alpha} \mathrm{C}_{\alpha \alpha^{\prime}}(0)(\widetilde{\mathbf{E}} \cdot \mathbf{v})_{\alpha^{\prime}} \Delta f_{\alpha^{\prime}}^{\mathrm{eq}}-e\langle\widetilde{\mathbf{E}} \cdot \mathbf{v} h\rangle .
$$


In the second step we take the one-point spectral function $S_{f}$ in the static limit, substituting for $\Delta f$ from equation (12) in the right-hand side of equation (32) to give

$$
S_{f}(\mathbf{r}, 0)=\frac{e^{2}}{\Omega(\mathbf{r})} \sum_{\mathbf{k}, s} \sum_{\mathbf{r}^{\prime}} \sum_{\mathbf{k}^{\prime}, s^{\prime}}(\widetilde{\mathbf{E}} \cdot \mathbf{v})_{\alpha} \mathrm{C}_{\alpha \alpha^{\prime}}(0)(\widetilde{\mathbf{E}} \cdot \mathbf{v})_{\alpha^{\prime}} \Delta f_{\alpha^{\prime}}^{\mathrm{eq}}+S_{g}(\mathbf{r}, 0),
$$

where $S_{g}(\mathbf{r}, 0)$ is generated by replacing $\Delta f$ with $\Delta g \equiv \Delta f-\Delta f^{\text {eq }}$ in equation (31), and subsequently in equation (32). Direct comparison of equations (35) and (36) leads to

$$
\frac{S_{f}(\mathbf{r}, 0)}{k_{\mathrm{B}} T}=P(\mathbf{r})+e\langle\widetilde{\mathbf{E}} \cdot \mathbf{v} h\rangle+\frac{S_{g}(\mathbf{r}, 0)}{k_{\mathrm{B}} T} .
$$

This is the precise connection between the non-equilibrium thermal current fluctuations and resistive dissipation in the system.

The limiting weak-field form of equation (37) is easily obtained. We prove that it is the linear fluctuation-dissipation theorem. Observe that the term in $h$ on the right-hand side varies as $\widetilde{E} g^{2}$, while the final term varies as $\widetilde{E}^{2} \Delta g$; both of these contributions are therefore of order $\widetilde{E}^{3}$. Suppose that the system is uniform. Then $\widetilde{\mathbf{E}}=\mathbf{E}_{\text {ext }}=\mathbf{E}$ acts along the $x$-axis. Division by $E^{2}$ on both sides of equation (37) gives

$$
\frac{1}{E^{2}} \frac{S_{f}(\mathbf{r}, 0)}{k_{\mathrm{B}} T} \rightarrow \frac{\left|J_{x}\right|}{E}=\sigma
$$

where $\sigma$ is the low-field conductivity. Equation (38) is the canonical FDT.

The non-dissipative and purely non-equilibrium structures beyond $P(\mathbf{r})$ can be expanded similarly to it. We discuss the symmetric-scattering case, for which there is no contribution $e\langle\widetilde{\mathbf{E}} \cdot \mathbf{v} h\rangle$. Within $S_{g}$ we apply the formula for the adiabatic propagator, equation (27), to express $\Delta g=\sum G \Delta f^{\mathrm{eq}}$ in terms of the correlated dynamic response function $\mathrm{C}(\omega)$. This produces the closed form

$$
\begin{aligned}
S_{g}(\mathbf{r}, 0) & =\frac{e^{2}}{\Omega(\mathbf{r})} \sum_{\mathbf{k}, s} \sum_{\beta}(\widetilde{\mathbf{E}} \cdot \mathbf{v})_{\alpha} \mathrm{C}_{\alpha \beta}(0)(\widetilde{\mathbf{E}} \cdot \mathbf{v})_{\beta}\left(\sum_{\alpha^{\prime}} \mathrm{C}_{\beta \alpha^{\prime}}(0) \frac{e \widetilde{\mathbf{E}}\left(\mathbf{r}^{\prime}\right)}{\hbar} \cdot \frac{\partial \Delta f_{\alpha^{\prime}}^{\text {eq }}}{\partial \mathbf{k}^{\prime}}\right) \\
& =-\frac{e^{3}}{k_{\mathrm{B}} T} \frac{1}{\Omega(\mathbf{r})} \sum_{\mathbf{k}, s} \sum_{\alpha^{\prime}}(\widetilde{\mathbf{E}} \cdot \mathbf{v})_{\alpha}(\mathrm{C}(0) \widetilde{\mathbf{E}} \cdot \mathbf{v})_{\alpha \alpha^{\prime}}^{2}\left(1-2 f_{\alpha^{\prime}}^{\mathrm{eq}}\right) \Delta f_{\alpha^{\prime}}^{\text {eq }} .
\end{aligned}
$$

The second line follows from the first after using equation (33) to express $\partial \Delta f^{\text {eq }} / \partial \mathbf{k}$ in terms of $f^{\text {eq }}$ and $\Delta f^{\text {eq }}$, and taking an inner sum into $(\mathrm{C}(0) \widetilde{\mathbf{E}} \cdot \mathbf{v})^{2}$.

The expression above differs markedly from the rate of energy loss $P(\mathbf{r})$ by Joule heating. In contrast, $S_{g}(\mathbf{r}, 0)$ relates directly to non-equilibrium broadening of the fluctuations, due to the excess energy gained from the field during intervals of ballistic flight [27]. The extent of the broadening is limited by dynamical dissipation of the excess energy, locally (by prompt inelastic scattering) or remotely (by carrier relaxation in the ideally absorbing terminals). The impact of this term on current noise is felt only for substantial departures from the weak-field regime.

There exist several alternative generalizations of the FDT for extended bulk systems 43 45]. We mention the best known, which defines the non-equilibrium noise temperature $T_{\mathrm{n}}$ pivotal to the interpretation of device-noise data [44. Phenomenologically $T_{\mathrm{n}}$ is obtained, for a non-linear operating point, by normalizing $S_{f}$ with the differential conductivity $\sigma_{x}(\widetilde{E})=\partial J_{x} / \partial \widetilde{E}_{x}$ such that $k_{\mathrm{B}} T_{\mathrm{n}}(\widetilde{E}) \equiv S_{f} / \sigma_{x}(\widetilde{E}) \widetilde{E}_{x}^{2}$, corresponding to the output of a small-signal noise measurement. (In general $T_{\mathrm{n}}$ is not isotropic.) Our equations (35) - 39 provide a microscopic framework for computing the noise spectral density in a wide class of degenerate systems. Since $\sigma_{x}(\widetilde{E})$ is also calculable within the same framework, this yields $T_{\mathrm{n}}$.

\section{APPLICATION TO HIGH-FIELD NOISE}

We can now explore one of the most significant properties of the excess spectrum $S_{g}$ : its strong inhibition by degeneracy. That there exists an additional, purely quantum-statistical, constraint on field-driven broadening is seen directly in the factor $\left(1-2 f^{\mathrm{eq}}\right)$ of equation (39). This suppresses the contribution of $S_{g}$ relative to the corresponding classical result, in which the factor is unity. Suppression of electron heating by Pauli exclusion reflects the large energy cost of displacing electrons deep inside the Fermi sea. 
To highlight the difference between dissipative and hot-electron terms, we revisit a simple example [35,46], the uniform electron gas in the constant-collision-time (Drude) approximation subject to a field $\mathbf{E}=-E \hat{\mathbf{x}}$. Expressions for the power density $P$ and hot-electron component $S_{g}$ are derived in the Appendix. The thermally driven currentcurrent spectral density, taken over a uniform sample of length $L_{x}$ and total volume $\Omega$, is given by 44]

$$
\begin{aligned}
\mathcal{S}(E, \omega) & \stackrel{\text { def }}{=} 4 \sum_{\mathbf{r}} \Omega(\mathbf{r}) \sum_{\mathbf{r}^{\prime}} \Omega\left(\mathbf{r}^{\prime}\right)\left\langle\left\langle\left(-\frac{e v_{x}}{L_{x}}\right)\left(-\frac{e v_{x}^{\prime}}{L_{x}}\right) \Delta \mathrm{f}^{(2)}(\omega)\right\rangle\right\rangle_{\mathrm{c}}^{\prime} \\
& =4 \frac{\Omega S_{f}(\omega)}{L_{x}^{2} E^{2}} .
\end{aligned}
$$

Writing the sample conductance as $\mathcal{G}=\Omega P / L_{x}^{2} E^{2}$, the static limit of the spectrum is determined by equation (37):

$$
\mathcal{S}(E, 0)=4 \mathcal{G} k_{\mathrm{B}} T\left[1+\frac{S_{g}(0)}{P k_{\mathrm{B}} T}\right]=4 \mathcal{G} k_{\mathrm{B}} T\left[1+\frac{\Delta n}{n}\left(\frac{m^{*} \mu_{\mathrm{e}}^{2} E^{2}}{k_{\mathrm{B}} T}\right)\right] .
$$

We have substituted for $P$ and $S_{g}$ respectively from equations (A7) and A9). The electronic density is $n$ while $\Delta n=\Delta N / \Omega$ is the number-fluctuation density. The effective electron mass is $m^{*}$ and $\mu_{\mathrm{e}}$ is the mobility.

The term $S_{g} / P k_{\mathrm{B}} T$ is a relative measure of the hot-electron contribution to the noise. The inhibiting effect of degeneracy, through $\Delta n / n$, is greatest at low temperature and least in the classical regime. When the Fermi energy $\varepsilon_{\mathrm{F}}$ satisfies $\varepsilon_{\mathrm{F}} \ll k_{\mathrm{B}} T$, the ratio $\Delta n / n$ goes to unity and the hot-electron term is that of a classical electron gas (low density, high temperature). Its form in the high-field limit $E \gg \sqrt{k_{\mathrm{B}} T / m^{*} \mu_{\mathrm{e}}^{2}}$ is $\mathcal{S} \sim 4 \mathcal{G} m^{*} \mu_{\mathrm{e}}^{2} E^{2}$, asymptotically independent of $T$.

On the other hand, when $k_{\mathrm{B}} T \ll \varepsilon_{\mathrm{F}}$ the system is strongly degenerate. In a $\nu$-dimensional system we have $\varepsilon_{\mathrm{F}} \propto n^{2 / \nu}$. Then

$$
\frac{\Delta n}{n}=\frac{k_{\mathrm{B}} T}{n} \frac{\partial n}{\partial \varepsilon_{\mathrm{F}}} \rightarrow \frac{\nu k_{\mathrm{B}} T}{2 \varepsilon_{\mathrm{F}}}
$$

with equation (41) this leads to

$$
\frac{\mathcal{S}(E, 0)}{\mathcal{S}(0,0)} \rightarrow 1+\frac{\nu}{2}\left(\frac{m^{*} \mu_{\mathrm{e}}^{2} E^{2}}{\varepsilon_{\mathrm{F}}}\right)
$$

Note that the thermal fluctuation spectrum $\mathcal{S}(E, 0)$ necessarily vanishes with temperature, while its ratio with the Johnson-Nyquist spectral density $\mathcal{S}(0,0)=4 \mathcal{G} k_{\mathrm{B}} T$ continues to exhibit a hot-electron excess which is now scaled by the Fermi energy.

Figure 1 displays the excess-noise spectral ratio in a two-dimensional electron gas, as a function of the applied field, when $T$ ranges from the degenerate limit to well above the Fermi temperature $T_{\mathrm{F}}=\varepsilon_{\mathrm{F}} / k_{\mathrm{B}}$. For $T$ much greater than both $T_{\mathrm{F}}$ and $m^{*} \mu_{\mathrm{e}}^{2} E^{2} / k_{\mathrm{B}}$ the excess contribution becomes classical, independent of temperature, and thus small compared with the now-dominant base value $\mathcal{S}(0,0)$. This is evident in figure 1 through the gradual downward shift of the plots, with increasing $T$.

Equation (42) may be compared with a perturbative estimate by Landauer 477] in the degenerate limit, for which the analogous excess term is $\left(\delta U / k_{\mathrm{B}} T\right)^{2}$, where $\delta U \sim m^{*} \mu_{\mathrm{e}} E v_{\mathrm{F}}$ is a characteristic energy gain. Taken at face value, this would suggest that hot-electron effects in the low- $T$ regime can be enhanced even more by further cooling of the system.

This counter-intuitive result comes from inappropriate use of perturbation analysis. Series expansion of the thermal current noise, in powers of $E$, fails to account for non-analyticity of the full non-perturbative solution in its approach to equilibrium [48]. Non-analyticity of the distribution function $f_{\mathbf{k}}$ precludes the reliable calculation of moment averages by expanding about equilibrium, as in reference 47]. (It is reassuring - and only seemingly fortuitous - that the actual linear current response is reproduced exactly by solving the transport equation, as usual, to first order in the field 27.)

The relevance of non-analyticity to transport physics has been questioned by Kubo, Toda, and Hashitsume [49]. They regard its appearance as spurious, a specific artefact of the crude way in which the Drude approximation treats real collisions. That is to overlook the appreciably broader evidence for non-analyticity in the variety of collision models assembled by Bakshi and Gross [48].

Even within the Drude model of a degenerate conductor (over-simplified though it is), it is clear that its exact nonperturbative solution does produce physically consistent scaling of the excess noise with $T$. Equally clearly, finite-order response theory does not. Kubo linear response recovers only $\mathcal{S}(0,0)$ and misses the non-linear excess noise altogether. 
Such sharp differences between perturbative and non-perturbative predictions should be experimentally measurable in the hot-electron spectrum. In our view, issues of non-analyticity and its physical manifestation remain open.

We make some final comments on shot noise and the impossibility 20,21.30 of a theoretical cross-over, unifying thermal and shot noise for mesoscopic metallic wires. The diffusive cross-over formula, ostensibly identical in form to equation (40), always generates a $T$-independent term [12 16]. One might have expected that a computation of the spectral density of equation (40), taken in the semi-classical quasi-ballistic limit $L_{x} \ll m^{*} v_{\mathrm{F}} \mu_{\mathrm{e}} / e$, would yield an expression for $\mathcal{S}(E, 0)$ that is independent of $T$ and proportional to the current $I=\mathcal{G} V$; in other words, shot noise [27].

We have carried out this quasi-ballistic exercise for a degenerate system, in simplified form [46]. At high fields it gives $\mathcal{S}(E, 0) \sim 2 e I\left(k_{\mathrm{B}} T / \varepsilon_{\mathrm{F}}\right)$. This is indeed linear in $I$ but thermal nevertheless, since its immediate source is the generic spectral relation, equation (39).

Thermal fluctuations are induced by spontaneous and quasi-continuous changes in the total internal energy of carriers, throughout the whole active volume of a device. Shot-noise fluctuations are induced by spontaneous and discrete changes in total carrier number, through the device's interfaces with the outer circuit. Such qualitative and topological distinctions may be of little practical importance in the classical macroscopic world. However, it is not at all clear that they are immaterial to the metallic mesoscopic regime. The issue is under active examination [30].

A leading task is to identify the kinetic origin of the empirical cross-over between thermal and shot noise, apparent in real mesoscopic conductors [1 5 . Once again we stress that, regardless of how shot noise is to be described microscopically, the logical and conceptual gaps between diffusive explanations of the cross-over (quantum as well as semi-classical) 9, 17,31] and strictly conventional kinetic theory have already been uncovered, characterized and analyzed [20,21]. We will present a fully detailed semi-classical kinetic model of shot noise in due course.

\section{SUMMARY}

We have described, and applied, a genuinely non-equilibrium kinetic formalism for current fluctuations. It holds for metallic systems down to mesoscopic scales, within the ambit of semi-classical theory. Our strategy for incorporating microscopic Fermi-liquid correlations within the Boltzmann picture safeguards the conservation laws at the two-body level. Conservation continues to underpin the nature of current noise at high fields.

Our theory leads to a precise quantitative link between non-equilibrium thermal current fluctuations and energy dissipation. In its low-field form, this is the standard fluctuation-dissipation theorem of linear-response analysis. At high fields, it highlights the pervasiveness of strong degeneracy even in hot-electron noise.

In a completely standard description, such as ours, correctness and calculability do not issue from the $a d$ hoc assumption of fictive Langevin noise sources; even less do they rely on diffusive analogies that fail manifestly to respect the canonical sum rules in electronic Fermi systems. Rather, the model's integrity will stem from the microscopic structure of its underlying Green functions. They describe how the fundamental electron-hole pair excitations evolve within a metallic conductor, in the semi-classical limit.

We have discussed how to map these native polarized correlations non-perturbatively, from their equilibrium distribution to its analogue in the externally driven conductor. The resulting high-field noise spectrum yields a faithful signature of its source: the elementary non-equilibrium electron-hole polarization processes.

The main, and physically inevitable, consequence of this rigidly orthodox kinetic investigation is the intrinsic scaling of degenerate-electron fluctuations with thermodynamic temperature. For diffusive phenomenologies, this is one phenomenon too many. To sustain their predictions for the shot noise of metallic wires, they have no choice but to deny outright all possibility of $T$-scaling for hot-electron noise [21]. This must be so for any theory that predicts a seamless cross-over between thermal and shot noise.

There is an undeniable connection between $T$-scaling and the dominance of long-range screening, as of degeneracy, in the polarizable electron gas. Logical examination shows that this nexus can be broken only by contradicting the standard picture of charge fluctuations in metals. Diffusively inspired models would seem to do exactly that [19 21]. So far, no such model has rationalized the heroic departure from principles that have been understood, widely and thoroughly, for some time 32].

We envisage two extensions to this work: the systematic inclusion of Coulomb screening within the microscopic structure of the fluctuations [29], and the analysis of shot noise as a kinetic process quite separate from thermally driven noise [30]. Coulomb effects are particularly evident in strongly confined electron systems, such as the twodimensional electron gas in a III-V heterojunction quantum well [28]. Self-consistent Coulomb screening in a confined channel should markedly reduce the scale of thermal fluctuations in the current.

Shot noise and thermal noise have disparate properties, which no-one disputes. Shot noise never scales with ambient temperature, while excess thermal current noise must do so if there is strong degeneracy. Coulomb effects too may 
differentiate between the two kinds of fluctuations. If so, then selective action of the Coulomb correlations could serve as an experimental tool to distinguish between excess thermal current noise and shot noise. This would help to pin-point both the distinct sources of non-equilibrium mesoscopic fluctuations and the disposition of Coulomb forces at small scales. We take up these themes in the forthcoming papers.

Every formalism for mesoscopic noise stands or falls by its new predictions. Ours is no exception 20,29]. Boltzmannian kinetics are obviously not equipped to give the final word on quantum fluctuation effects; be that as it may, it hardly needs saying that any mesoscopic model, whatever its origin, should be totally consistent with the established physical facts. In the context of the metallic electron gas, noise descriptions which claim to be truly microscopic must address full sum-rule consistency as a matter of course. This transcends semi-classical analysis and is by far our most important message.

\section{ACKNOWLEDGMENTS}

We are indebted to the late $\mathrm{R}$ Landauer for his generous encouragement in the preliminary stages of this work. We thank Erika Davies for help with calculations and figures and N W Ashcroft, R J-M Grognard, K I Golden and D Neilson for fruitful discussions.

\section{APPENDIX A: UNIFORM DRUDE MODEL}

We derive the dynamical fluctuation structure for a single parabolic conduction band with uniform electron density $n$ and constant mobility $\mu_{\mathrm{e}}=e \tau / m^{*}$, where $\tau$ is the spin-independent collision time and $m^{*}$ the effective mass. The system is driven by a uniform field $\widetilde{\mathbf{E}}=\mathbf{E}=-E \hat{\mathbf{x}}$ acting in the negative (drain to source) direction. We take variations which are homogeneous over the sample region, so that the fluctuations of interest have no spatial dependence.

The Boltzmann equation in the model is

$$
\left[\frac{\partial}{\partial t}+\frac{e E}{\hbar} \frac{\partial}{\partial k_{x}}+\frac{1}{\tau}\right] f_{\mathbf{k}}(t)=\frac{\langle f(t)\rangle}{\left\langle f^{\mathrm{eq}}\right\rangle} \frac{f_{k}^{\mathrm{eq}}}{\tau} .
$$

Since the Boltzmann operator is linear, the fluctuation structure is qualitatively similar to that for elastic scattering [differences arise from the inhomogeneous term in $f^{\mathrm{eq}}$, notably in the behaviours of $R(t)$ and $\Delta f(t)$ ]. We solve equation (A1) by Fourier transforms in reciprocal space, so that the transform $F_{\boldsymbol{\rho}} \equiv \Omega^{-1} \sum_{\mathbf{k}} f_{\mathbf{k}} \exp (i \mathbf{k} \cdot \boldsymbol{\rho})$ of the steady-state distribution takes the form

$$
F_{\rho}=\frac{F_{\mathbf{0}}}{F_{0}^{\mathrm{eq}}} \frac{F_{\rho}^{\mathrm{eq}}}{1-i k_{d} \rho_{x}}
$$

where $k_{d}=e E \tau / \hbar$ and $F_{\mathbf{0}}=\frac{1}{2}\langle f\rangle$ per spin state. While a formal distinction is made between $F_{\mathbf{0}}$ and $F_{0}^{\text {eq }}$, the physical normalization is always $F_{\mathbf{0}}=F_{0}^{\mathrm{eq}}=\frac{1}{2} n$. Note also that $F_{\boldsymbol{\rho}}$ is singular for $\rho_{x}=-i k_{d}^{-1}$. In wave-vector space this means that $f_{\mathbf{k}}$ is non-analytic at $E=0$. The same can be said for $\Delta f_{\mathbf{k}}$.

The transform of the dynamic response function,

$$
\mathcal{R}_{\boldsymbol{\rho} \boldsymbol{\rho}^{\prime}}(\omega) \equiv \frac{1}{\Omega^{2}} \sum_{\mathbf{k}} \sum_{\mathbf{k}^{\prime}} \mathbf{R}_{\mathbf{k k}^{\prime}}(\omega) \exp \left[i\left(\mathbf{k} \cdot \boldsymbol{\rho}-\mathbf{k}^{\prime} \cdot \boldsymbol{\rho}^{\prime}\right)\right]
$$

has the equation

$$
\left[-i \omega \tau-i k_{d} \rho_{x}+1\right] \mathcal{R}_{\boldsymbol{\rho} \boldsymbol{\rho}^{\prime}}(\omega)=\tau \delta\left(\boldsymbol{\rho}-\boldsymbol{\rho}^{\prime}\right)+\frac{\mathcal{R}_{\mathbf{0} \boldsymbol{\rho}^{\prime}}(\omega)}{F_{0}^{\mathrm{eq}}} F_{\rho}^{\mathrm{eq}}
$$

For $\boldsymbol{\rho}=\mathbf{0}$ this leads to

$$
\mathcal{R}_{\mathbf{0} \boldsymbol{\rho}^{\prime}}(\omega)=-\frac{\delta\left(\boldsymbol{\rho}^{\prime}\right)}{i(\omega+i \eta)}
$$

On the other hand, the low-frequency adiabatic part of $\mathcal{R}_{\boldsymbol{\rho} \rho} \boldsymbol{\rho}^{\prime}$ scales with the steady-state solution $F_{\boldsymbol{\rho}}$ [in a collisiontime model the asymptotic form $F_{\rho} / \frac{1}{2} n$ replaces $\left.\Delta F_{\rho} / \frac{1}{2} \Delta n\right]$. On denoting the correlated part by $\mathcal{C}_{\boldsymbol{\rho} \rho} \boldsymbol{\rho}^{\prime}$ and recalling that the adiabatic part exhausts the normalization of $\mathcal{R}_{\mathbf{0} \boldsymbol{\rho}^{\prime}}$, we obtain 


$$
\mathcal{R}_{\rho \rho^{\prime}}(\omega)=\mathcal{C}_{\boldsymbol{\rho} \rho^{\prime}}(\omega)-\frac{\delta\left(\boldsymbol{\rho}^{\prime}\right)}{i(\omega+i \eta)} \frac{F_{\boldsymbol{\rho}}}{F_{\mathbf{0}}}
$$

When the above is put together with equations A2 - (A4) we arrive, after some algebra, at the explicit formula for the correlated propagator:

$$
\mathcal{C}_{\boldsymbol{\rho} \boldsymbol{\rho}^{\prime}}(\omega)=\tau \frac{\delta\left(\boldsymbol{\rho}-\boldsymbol{\rho}^{\prime}\right)-\frac{F_{\boldsymbol{\rho}}}{F_{\mathbf{0}}} \delta\left(\boldsymbol{\rho}^{\prime}\right)}{1-i k_{d} \rho_{x}-i \omega \tau} .
$$

We can use equation (A6) directly to evaluate both dissipative and non-dissipative contributions to the noise. Using the reciprocal-space representation $\mathbf{v} \leftrightarrow-i\left(\hbar / m^{*}\right) \partial / \partial \boldsymbol{\rho}$, the power density $P$ of equation (35) is

$$
\begin{aligned}
P & =2 \frac{e^{2} E^{2}}{k_{\mathrm{B}} T}\left(-\frac{i \hbar}{m^{*}}\right)^{2}\left\{\frac{\partial}{\partial \rho_{x}} \int d^{\nu} \rho^{\prime} \mathcal{C}_{\boldsymbol{\rho} \rho^{\prime}}(0) \frac{\partial}{\partial \rho_{x}^{\prime}} \Delta F_{\rho^{\prime}}^{\mathrm{eq}}\right\}_{\rho \rightarrow 0} \\
& =2 \frac{e^{2} E^{2} \tau}{k_{\mathrm{B}} T}\left(\frac{\hbar}{m^{*}}\right)^{2}\left\{-\frac{\partial^{2}}{\partial \rho_{x}^{2}} \Delta F_{\rho}^{\mathrm{eq}}\right\}_{\rho \rightarrow 0} \\
& =\sigma E^{2} .
\end{aligned}
$$

The Drude conductivity $\sigma=n e \mu_{\mathrm{e}}$ appears when we apply the relation

$$
\left\{-\frac{\partial^{2}}{\partial \rho_{x}^{2}} \Delta F_{\rho}^{\mathrm{eq}}\right\}_{\rho \rightarrow 0}=\left\langle k_{x}^{2} \Delta f^{\mathrm{eq}}\right\rangle=\frac{m^{*} k_{\mathrm{B}} T}{\hbar^{2}}\left(\frac{n}{2}\right)
$$

to the middle line of the equation. A contribution containing $\left\langle v_{x} \Delta f^{\mathrm{eq}}\right\rangle=0$ vanishes trivially.

The hot-electron spectral density $S_{g}$ in the static limit [recall equation (39)] is calculated similarly:

$$
\begin{aligned}
S_{g}= & 2 \frac{(e \mathbf{E} \cdot \hat{\mathbf{x}})^{3}}{\hbar}\left\{\int d^{\nu} \rho^{\prime} \int d^{\nu} \rho^{\prime \prime} v_{x} \mathcal{C}_{\boldsymbol{\rho} \boldsymbol{\rho}^{\prime}}(0) v_{x}^{\prime} \mathcal{C}_{\boldsymbol{\rho}^{\prime} \boldsymbol{\rho}^{\prime \prime}}(0)\left(-i \rho_{x}^{\prime \prime} \Delta F_{\rho^{\prime \prime}}^{\mathrm{eq}}\right)\right\}_{\rho \rightarrow 0} \\
= & 2 \frac{e^{3} E^{3} \tau^{2} \hbar}{m^{* 2}}\left\{\left[\frac{\partial}{\partial \rho_{x}} \frac{1}{1-i k_{d} \rho_{x}}\left(\frac{\partial}{\partial \rho_{x}} \frac{-i \rho_{x} \Delta F_{\rho}^{\mathrm{eq}}}{1-i k_{d} \rho_{x}}\right)\right]_{\rho \rightarrow 0}\right. \\
& \left.-\left[\frac{\partial}{\partial \rho_{x}} \frac{F_{\boldsymbol{\rho}} / F_{\mathbf{0}}}{1-i k_{d} \rho_{x}}\right]_{\rho \rightarrow 0}\left[\frac{\partial}{\partial \rho_{x}^{\prime}} \frac{-i \rho_{x}^{\prime} \Delta F_{\rho^{\prime}}^{\mathrm{eq}}}{1-i k_{d} \rho_{x}^{\prime}}\right]_{\rho^{\prime} \rightarrow 0}\right\} .
\end{aligned}
$$

We evaluate this with the help of the relations $\Delta F_{0}^{\mathrm{eq}}=\frac{1}{2} \Delta n$ and $\left\{\partial F_{\boldsymbol{\rho}} / \partial \rho_{x}\right\}_{\rho \rightarrow 0}=i k_{d} F_{\mathbf{0}}$, the latter following from equation (A2). The result is

$$
S_{g}=\sigma m^{*} \mu_{\mathrm{e}}^{2} E^{4}\left(\frac{\Delta n}{n}\right)
$$

[1] Liefrink F, Dijkhuis J I, de Jong M J M, Molenkamp L W and van Houten H 1994 Phys. Rev. B 4914066

[2] Reznikov M, Heiblum M, Shtrikman H and Mahalu D 1995 Phys. Rev. Lett. 753340

[3] Kumar A, Saminadayar L, Glattli D C, Jin Y and Etienne B 1996 Phys. Rev. Lett. 762778

[4] Steinbach A H, Martinis J M and Devoret M H 1996 Phys. Rev. Lett. 763806

[5] Schoelkopf R J, Burke P J, Kozhevnikov A A, Prober D E and Rooks M J 1997 Phys. Rev. Lett. 783370

[6] Pothier H, Guéron S, Birge N O, Esteve D and Devoret M H 1997 Phys. Rev. Lett. 793490

[7] de Picciotto R, Reznikov M, Heiblum M, Umansky V, Bunin G and Mahalu D 1997 Nature 389162

[8] Saminadayar L, Glattli D C, Jin Y and Etienne B 1997 Phys. Rev. Lett. 792526

[9] de Jong M J M and Beenakker C W J 1997 Mesoscopic Electron Transport (NATO ASI Series E) ed L P Kouwenhoven, G Schön and L L Sohn (Kluwer Academic, Dordrecht) 
[10] Khlus V A 1987 Sov. Phys. JETP 661243

[11] Lesovik G B 1989 JETP Lett. 49592

[12] Büttiker M 1992 Phys. Rev. Lett. 65 2901; 1992 Phys. Rev. B 4612485

[13] Beenakker C W J and Büttiker M 1992 Phys. Rev. B 46189

[14] Martin Th and Landauer R 1992 Phys. Rev. B 451742

[15] Nagaev K E 1992 Phys. Lett. A 169 103; 1995 Phys. Rev. B 524740

[16] de Jong M J M and Beenakker C W J 1995 Phys. Rev. B 5116867

[17] Blanter Ya and Büttiker M 1999 cond-mat/9910158.

[18] Datta S 1995 Electronic Transport in Mesoscopic Systems (Cambridge University Press, Cambridge)

[19] For example: the compressibility sum rule (see reference [32] below) links the magnitude of electron-hole correlations to the total density of a degenerate system. In the diffusive approaches, the correlations are perforce keyed to the notional density of mobile diffusers only, because the carriers deep in the Fermi sea are excluded from the calculation of diffusive transport [18] (moreover, this notional density is defined ad hoc). This leads directly to an unphysical screening response and violation of quasi-neutrality (perfect screening sum rule) over the size of the sample.

[20] Green F and Das M P 2000 Proceedings of the Second International Conference on Unsolved Problems of Noise and Fluctuations (UPoN'99) ed D Abbott and L B Kish AIP 511 (American Institute of Physics, New York) pp 422-33. For a similar discussion see cond-mat/9905086.

[21] Das M P and Green F 1999 in Proceedings of the 23rd International Workshop on Condensed Matter Theories ed G S Anagnostatos (Nova Science, in preparation); see also cond-mat/9910183

[22] Reggiani L, Reklaitis A, González T, Mateos J, Pardo D and Bulashenko O M 2000 Aust. J. Phys. 533

[23] Tadyszak P, Danneville F, Cappy A, Reggiani L, Varani L and Rota L 1996 Appl. Phys. Lett. 691450

[24] González T, Mateos J, Pardo D, Varani L and Reggiani L 1999 cond-mat/9910125

[25] Korman C E and Mayergoyz I D 1996 Phys. Rev. B 5417620

[26] Stanton C J and Wilkins J W 1987 Phys. Rev. B 35 9722; 1987 Phys. Rev. B 361686

[27] Stanton C J 1986 Ph.D. thesis (Cornell University, unpublished)

[28] Ferry D K and Goodnick S M 1997 Transport in Nanostructures (Cambridge University Press, Cambridge, UK)

[29] Green F and Das M P 2000 submitted to J. Phys. Condensed Matter. See also cond-mat/9911251

[30] For a preliminary account see Green F and Das M P 1998 cond-mat/9809339 (CSIRO-RPP3911, unpublished).

[31] Kogan Sh M 1996 Electronic Noise and Fluctuations in Solids (Cambridge University Press, Cambridge, UK)

[32] Pines D and Nozières P 1966 The Theory of Quantum Liquids (Benjamin, New York)

[33] Kogan Sh M and Shul'man A Ya 1969 Zh. Eksp. Teor. Fiz. 56862 [1969 Sov. Phys. JETP 29 467]

[34] Gantsevich S V, Gurevich V L and Katilius R 1979 Nuovo Cimento 21

[35] Green F and Chivers M J 1996 Phys. Rev. B 545791

[36] van Kampen N G 1981 Stochastic Processes in Physics and Chemistry (North-Holland, Amsterdam) pp 246-52

[37] The Poisson equation is always defined in three dimensions. To interpret equation (3a) appropriately when $\nu<3$, the electron density $\langle f\rangle$ must be understood to carry a (separable) factor in the $3-\nu$ transverse space co-ordinates. Thus, for a transport problem confined strictly to two dimensions, the Poisson source term contains $\langle f(\mathbf{r})\rangle \equiv \delta(z)\left\langle f\left(\mathbf{r}_{\perp}\right)\right\rangle$, where $z$ is orthogonal to the plane $\left(\mathbf{r}_{\perp} ; z=0\right)$. On the other hand, the stabilizing background distribution $n^{+}(\mathbf{r})$ can be fully three-dimensional, as in a modulation-doped heterostructure 28.

[38] The subtraction of left- and right-hand sides of equation (2) from (11) is formally necessary, despite the fact that both are identically zero. This is because we will later require their functional derivatives with respect to $f^{\text {eq }}$. Those variations do not vanish identically, as a quick test on equation (2) shows.

[39] We follow convention in taking the external field as primitive. It induces the system response to be described by transport theory, but is not itself describable at that level. A more complete way of incorporating electromotive forces into the physics of transport is given in Magnus W and Schoenmaker W 1998 J. Math. Phys. 396715.

[40] Green F 1996 Phys. Rev. B 544394

[41] Kadanoff L P and Baym G 1962 Quantum Statistical Mechanics (W A Benjamin, Reading, Massachusetts)

[42] Note: a remark in reference [40], that $\Delta f(t)$ is inherently time-dependent, holds only for collision-time approximations.

[43] Van Vliet C M 1994 IEEE Trans. Electron Devices 411902

[44] Nougier J P 1980 Physics of Nonlinear Transport in Semiconductors ed D K Ferry, J R Barker, and C Jacoboni (Plenum, New York) p $415 \mathrm{ff}$

[45] Reggiani L, Lugli P and Mitin V 1988 Phys. Rev. Lett. 60736

[46] Green F and Das M P 1998 Recent Progress in Many-Body Theories ed D Neilson and R F Bishop (World Scientific, Singapore) p. 102. See also cond-mat/9709142

[47] Landauer R 1993 Phys. Rev. B 4716427

[48] Bakshi P M and Gross E P 1968 Ann. Phys. 24419

[49] Kubo R, Toda M and Hashitsume N 1991 Statistical Physics II: Nonequilibrium Statistical Mechanics (2nd ed., Springer, Berlin) pp 199 and 200 
FIG. 1. Zero-frequency spectral density of the excess (hot-electron) thermal noise, above the equilibrium noise, in a degenerate and uniform two-dimensional electron gas. The excess is plotted as its ratio with the zero-field noise $\mathcal{S}(E=0)=4 \mathcal{G} k_{\mathrm{B}} T$ as a function of driving field $E$ and for temperatures $T$ between 0 and $900 \mathrm{~K}$, in steps of $150 \mathrm{~K}$. The dot-dashed line is at $T=300$ K. In the degenerate limit $T \ll T_{\mathrm{F}}$, thermal noise scales with $T$; thus the excess-noise ratio is independent of temperature. At high temperature, the excess ratio for a given field value diminishes as its denominator $\mathcal{S}(0)$ becomes dominant. 


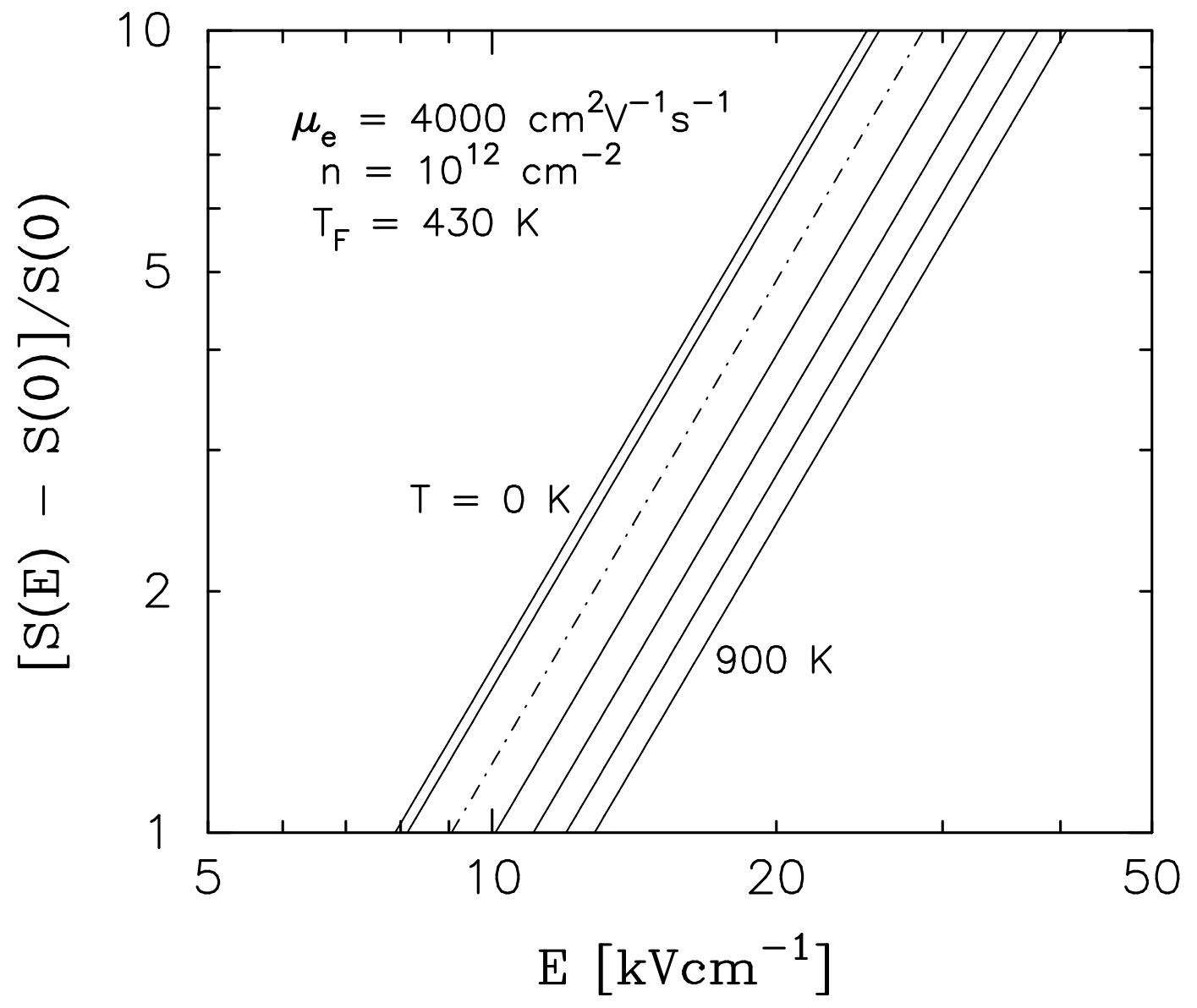

